\title{
The Interaction of Planets and Brown Dwarfs with AGB Stellar Winds
}

\author{
Hyosun $\mathrm{Kim}^{*}$ and Ronald E. Taam ${ }^{\dagger, *}$ \\ *Academia Sinica Institute of Astronomy and Astrophysics, P.O. Box 23-141, Taipei 10617, \\ Taiwan; hkim@asiaa.sinica.edu.tw \\ $\dagger$ Department of Physics and Astronomy, Northwestern University, 2131 Tech Drive, Evanston, IL \\ 60208; taam@tonic.astro.northwestern.edu
}

\begin{abstract}
Beyond the main sequence solar type stars undergo extensive mass loss, providing an environment where planet and brown dwarf companions interact with the surrounding material. To examine the interaction of substellar mass objects embedded in the stellar wind of an asymptotic giant branch (AGB) star, three dimensional hydrodynamical simulations at high resolution have been calculated utilizing the FLASH adaptive mesh refinement code. Attention is focused on the perturbation of the substellar mass objects on the morphology of the outflowing circumstellar matter. In particular, we determine the properties of the resulting spiral density wake as a function of the mass, orbital distance, and velocity of the object as well as the wind velocity and its sound velocity. Our results suggest that future observations of the spiral pattern may place important constraints on the properties of the unseen low mass companion in the outflowing stellar wind.
\end{abstract}

Keywords: binaries: general — hydrodynamics — stars: AGB and post-AGB — stars: circumstellar matter — stars: late-type — stars: winds, outflows — waves

PACS: $97.10 . \mathrm{Fy}$, 95.75.Pq, 95.30.Lz

\section{INTRODUCTION}

Evolved giant stars lose a significant amount of mass in the form of stellar winds, providing material in regions for which orbiting planets or brown dwarfs interact with their immediate surroundings. The mass loss rate of these giant stars, especially during the asymptotic giant branch (AGB) phase of the stellar evolution, ranges from $10^{-7}$ to $10^{-3} M_{\odot} \mathrm{yr}^{-1}$ with a wind speed of $10-30 \mathrm{~km} \mathrm{~s}^{-1}$ (Fong et al. 2006). Such rates lead to an envelope density at $\sim 100$ AU distance from the stellar center of $10^{-19}-10^{-15}$ $\mathrm{g} \mathrm{cm}^{-3}$, approaching the typical density of protoplanetary disks (Hayashi 1981; Aikawa \& Herbst 1999) where planets form and grow by gathering the surrounding matter (e.g., Alibert et al. 2005; Hubickyj et al. 2005; Ikoma et al. 2000). The gravitational interaction between a protoplanet and its environmental disk has been investigated in great detail. Here, a spiral wave forms in the differentially rotating system (Goldreich \& Tremaine 1979; Masset 2008, and references therein), but the corresponding study in the context of systems interacting with an outflowing wind around the evolved giant stars is still lacking. Although the velocity direction of the background medium is the only difference between the rotating and outflowing systems, the entire background structure and the interaction aspects markedly differ. In the former case, where the surrounding matter is in the form of a differentially rotating disk, the background co-rotates locally with the substellar mass component of interest so that the torque at the resonance plays the crucial 
role in producing the spiral. However, in the case of a nearly spherically symmetric stellar wind, the motion of the substellar object is perpendicular to the background velocity field. The relative velocity of the background gas with respect to the substellar object causes a Bondi-Hoyle-Lyttleton (BHL) accretion column to lie behind the orbiting body (Bondi \& Hoyle 1944; Edgar 2004, and references therein).

Although starting from a different viewpoint, the accretion flow in a weak gravitational potential (so-called a density wake) is well formulated by a linear perturbation analysis in an initially uniform and static background (Ostriker 1999; Kim \& Kim 2007; Kim et al. 2008). In particular Kim \& Kim (2007) considered an object moving on a curvilinear orbit, which leads to a density wake in the form of an Archimedes spiral with the opening angle $\theta=\sin ^{-1}\left(c_{\mathrm{s}} / V_{p}\right)$, where $V_{p}$ and $c_{\mathrm{s}}$ represent the orbital and sonic speeds. In this contribution we show that the wind from the evolved central star pushes the wake outward in the radial direction so as to widen the opening of the spiral pattern. This provides the possibility for detecting the spiral pattern at large distances from the luminous central star and can be used to probe the presence of the unseen objects orbiting around the evolved star, if the density enhancement is sufficient. To predict the orbital properties of these substellar components from future observations of the spiral density wakes, we provide an empirical formula for the density enhancement in the spiral pattern. The reader is referred to Kim \& Taam (2011) for a more comprehensive discussion.

\section{SIMULATION SETUP}

Given a continuous stellar mass loss rate $\dot{M}_{*}$, a self-consistent configuration of density $\rho_{w}$ and velocity $\mathbf{V}_{\mathbf{w}}$ satisfies the steady hydrodynamic conditions

$$
\nabla \cdot\left(\rho_{w} \mathbf{V}_{\mathbf{w}}\right)=0
$$

and

$$
\mathbf{V}_{\mathbf{w}} \cdot \nabla \mathbf{V}_{\mathbf{w}}=-\frac{1}{\rho_{w}} \nabla P_{w}-\nabla \Phi_{*}
$$

for the gravitational potential of the central star $\Phi_{*}$ (Gail \& Sedlmayr 1987, Lamers \& Cassinelli 1999, see also Parker 1958 for the original description for isothermal wind solutions). To simplify the problem, we do not consider radiation pressure on dust coupled with the nearby gas, which is commonly regarded as the wind driving mechanism of the evolved stars (e.g., Winters et al. 2000). Although the driving mechanism affects the background wind condition, the response of gas to the gravitational perturbation due to the substellar object orbiting around the mass losing star is likely independent of the global wind structure as it is determined by the local wind quantities. This has been verified by performing two sets of simulations with transonic and supersonic branches of Parker's wind solution, confirming that the wake properties described here are determined by the wind density $\rho_{w}(r)$, its expansion speed $V_{w}(r)$, and the sound speed $c_{\mathrm{s}}(r)$ on the spot in addition to the orbital and accretion properties of the perturbing object.

We have investigated 42 hydrodynamic models for a range of object mass $M_{p}$, size $r_{s}$, orbital speed $V_{p}$, distance from the star $r_{p}$, as well as stellar mass loss rate $\dot{M}_{*}$, wind speed 

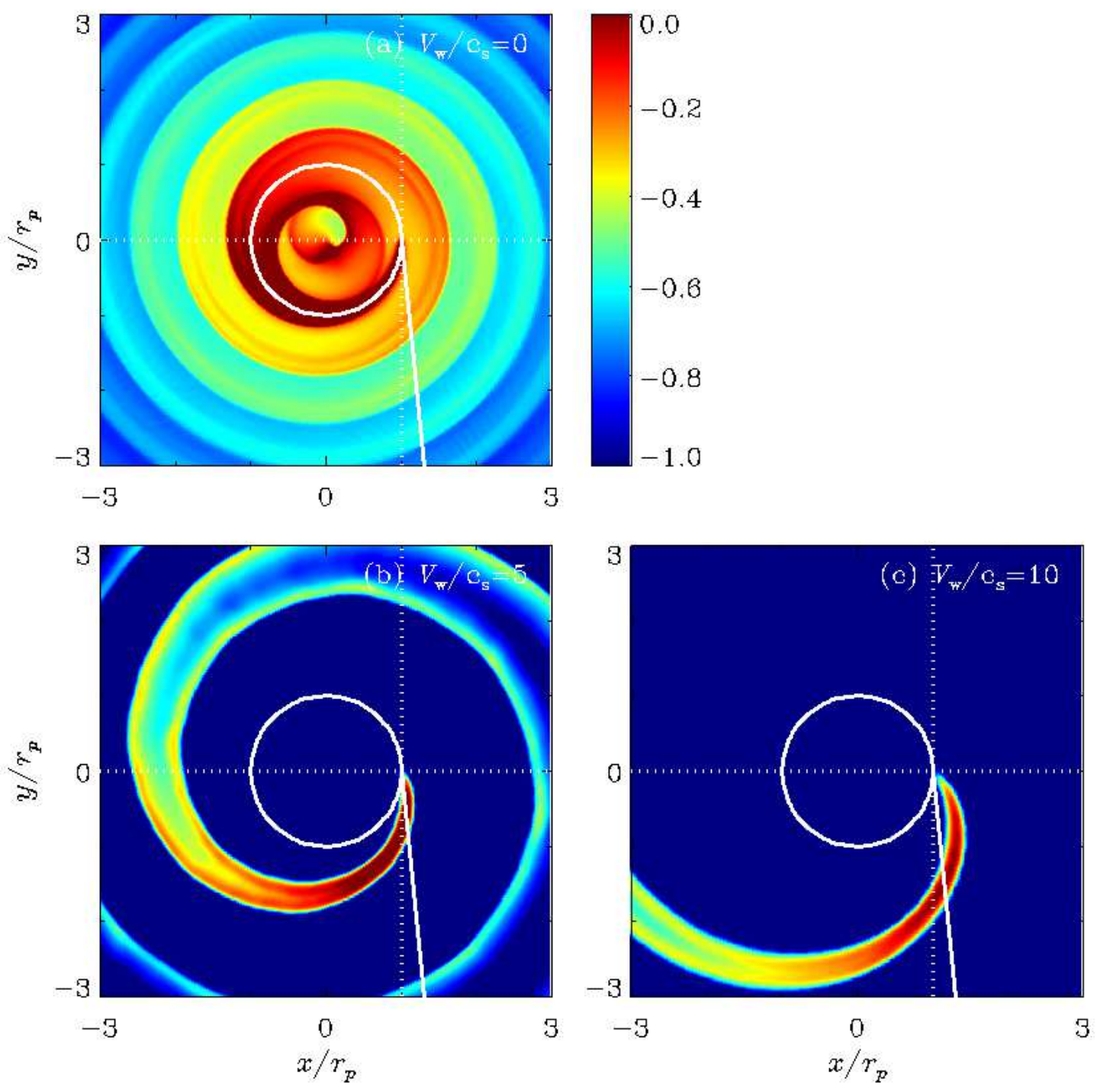

FIGURE 1. Comparison of perturbed density distribution between models with the background wind speed $V_{w}$ of (a) 0 , (b) 5, and (c) 10 in the units of sonic speed for a background gas characterized by $c_{\mathrm{s}}=3 \mathrm{~km} \mathrm{~s}^{-1}$. The perturbing object is modeled by an object of mass $M_{p}=0.1 M_{\odot}$ moving on a circular orbit at a distance $r_{p}=20 \mathrm{AU}$, marked by a white circle, in the counterclockwise direction with the orbital speed $V_{p}=10 c_{\mathrm{s}}$, currently located at $(x, y)=\left(r_{p}, 0\right)$. The white line denotes the opening angle of the spiral in the vicinity of the orbiting object in a static medium, $\theta=\sin ^{-1}\left(c_{\mathrm{s}} / V_{p}\right)$. The color bar labels the perturbed density in logarithmic scale.

$V_{w}$, and sound speed $c_{\mathrm{s}}$. Specifically, we have verified the dependence of density wake on three different velocities: $c_{\mathrm{s}}=3$ and $5 \mathrm{~km} \mathrm{~s}^{-1}, V_{p}$ is in the range of $0.5-10 c_{\mathrm{s}}$, and $V_{w}$ up to $10 c_{\mathrm{s}}$ satisfying the supersonic and transonic branches of isothermal wind models. The simulations are performed in three spatial dimensions, but here we only focus on the features in the orbital plane. See Kim \& Taam (2011) for the vertical structures. 


\section{SHAPE OF SPIRAL PATTERN}

The outflowing wind affects the shape of the spiral density wake, which is generated by the gravitational interaction of the substellar object with the background. As shown in Figure 1, the spiral wake pattern opens further with higher wind speeds. To show the difference of the opening angle of the spiral, one line is overlaid at the present position of the object, showing the angle, $\theta=\sin ^{-1}\left(c_{\mathrm{S}} / V_{p}\right)$, analytically calculated for the model in the absence of a wind (Ostriker 1999; Kim \& Kim 2007). This expression for the opening angle reveals that the pattern propagates in the radial direction with the sonic speed $c_{\mathrm{S}}$ as the perturbing object moves laterally with the speed $V_{p}$, i.e., the pattern propagation speed is $c_{\mathrm{S}}$ (Kim \& Kim 2007). Extending this picture to the outflowing models, one can glean from Figure 1 the wind speed is related to the pattern propagation speed, which is revealed in the opening angle of the spiral arm pattern.

Simple geometry yields the following relation

$$
\frac{d\left(r / r_{p}\right)}{d \varphi}=\frac{V_{a r m}}{V_{p}}
$$

from which the pattern propagation speed $V_{\text {arm }}$ relative to the orbital speed $V_{p}$ of the perturbing object can be derived from the shape of the spiral. Numerical differentiation at the shock boundary, exhibiting high density, gives the pattern propagation speed of $V_{w}+c_{\mathrm{s}}$, which is also predicted by linear analysis in the WKB approximation. Here, we point out that it is not well described purely by the speed of outflowing matter, which is often used by observers (e.g. Mauron \& Huggins 2006; Morris et al. 2006), especially when the wind speed is comparable to the thermal sound speed. Also it is to be noticed that the pattern is not necessarily an Archimedes spiral, in which the arm spacing is constant, but its shape possibly changes with the variation of wind speed and temperature as a function of distance.

\section{DENSITY ENHANCEMENT}

To detect the spiral arm pattern around the evolved giant stars, the density enhancement (or density jump) of the structure must exceed some threshold value. In our definition $\alpha=\rho / \rho_{w}-1$ represents this perturbed density normalized by the local background density $\rho_{w}(r)$, which has the profile close to $\sim r^{-2}$ in most areas.

Figure 2 exhibits the density enhancement for four different models with different combinations of the orbital speed and the wind speed but with a fixed sound speed, as well as the mass, radius, and orbital radius of the object, and the stellar mass and mass loss rate. We have also checked the effects of all other parameters enumerated here to obtain an empirical formula for the minimum value of the density peaks at the shock boundary of the spiral arm:

$$
\alpha_{\text {peak }} \gtrsim \frac{r_{B}}{\left|r-r_{p}\right|}\left(\frac{\frac{\left|r-r_{p}\right|}{r_{B}} V_{w}^{2}+10 V_{p} V_{w}+c_{\mathrm{s}}^{2}}{V_{w}^{2}+\left|V_{p}^{2}-c_{\mathrm{s}}^{2}\right|}\right)^{1 / 2} \quad \text { for } V_{w}>c_{\mathrm{s}},
$$



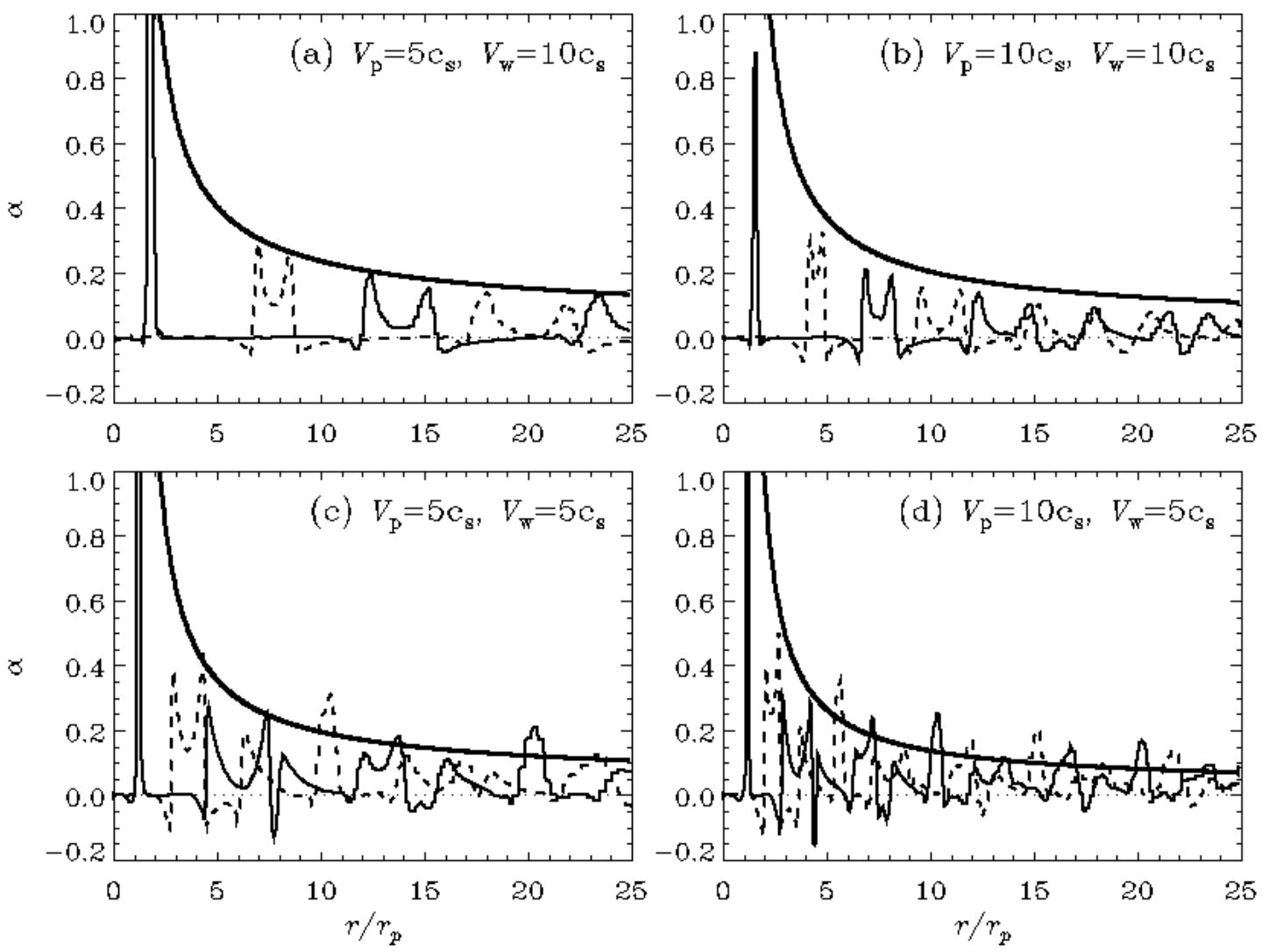

FIGURE 2. Density enhancement $\alpha$ of the spiral density wake formed by the gravitational interaction of the stellar wind with a substellar object of mass $0.1 M_{\odot}$ orbiting around the central star as a function of distance normalized by the orbital radius $r_{p}=20 \mathrm{AU}$. Comparison is achieved between the models with the orbital and wind speeds of (a) $V_{p}=5 c_{\mathrm{s}}, V_{w}=10 c_{\mathrm{s}}$, (b) $V_{p}=10 c_{\mathrm{s}}, V_{w}=10 c_{\mathrm{s}}$, (c) $V_{p}=5 c_{\mathrm{s}}, V_{w}=5 c_{\mathrm{s}}$, and (d) $V_{p}=10 c_{\mathrm{s}}, V_{w}=5 c_{\mathrm{s}}$, where the sound speed $c_{\mathrm{s}}$ is $3 \mathrm{~km} \mathrm{~s}^{-1}$. The lines denote the perturbed density profiles along the distance from the star in the direction of the object (solid) and in the opposite direction (dashed). See text for the complementary bold solid line that outlines the peaks.

where $r_{B}=G M_{p} / c_{\mathrm{s}}^{2}$ is the Bondi accretion radius. We mention that this empirical formula can be improved by exploring additional parameter space and/or by direct analytic approach, although it serves quite well for a large range of parameter space as the lower limit of density peaks that could be detected. Note that this equation is well checked only for the cases of a supersonic speed of wind, even though the density wakes in the transonic wind background appear reasonable in the inner subsonic regime.

For a fast wind, which is often observed in AGB envelopes, this equation (4) is reduced simply to

$$
\alpha_{\text {peak }} \gtrsim\left(\frac{r_{B}}{r}\right)^{1 / 2} \quad \text { for } V_{w} \gg V_{p}, c_{\mathrm{s}},
$$

when the observed arm is located at great distances from the central star relative to the orbital radius of the perturbing object. In comparison to the slow wind cases, the density peak profiles decrease with distance from the star with a power of -0.5 rather than -1 . 
This is favorable for observers seeking a signature of unseen low mass objects revolving about evolved mass losing giant stars.

\section{DISCUSSION}

\section{Prediction of the Orbital Properties of Planets and Brown Dwarfs Deduced from Spiral Structures of AGB Stellar Wind Envelopes}

Equation (5) indicates that for the typical envelope temperature of the cool giants, corresponding to the sound speed of $1-4 \mathrm{~km} \mathrm{~s}^{-1}$, the peak density jump for objects of $1 M_{J}, 30 M_{J}$, and $0.1 M_{\odot}$ is greater than 2.5-10\%, 14-55\%, and 25-100\%, respectively, at $100 \mathrm{AU}$ distance from the star. Although such enhancements are not large, they may be detectable in the near future with sufficiently sensitive observations. In the fast wind assumption, the mass of the unseen object may be estimated by measuring the density jump of the spiral arm at a given position, given knowledge of the sound speed. Furthermore, if more than one turn of the spiral is detected, the orbital properties could be also estimated by fitting the density peak profile.

In this case that more than one turn are detected, additional information can be inferred from the arm interval. Assuming the pattern propagation speed does not vary significantly along one turn, the integration of equation (3) gives the arm interval, defined by the distance between the outer boundary of the pattern, as following:

$$
\Delta r_{a r m}=\left(V_{w}+c_{\mathrm{s}}\right) \times \frac{2 \pi r_{p}}{V_{p}} .
$$

From this result, one can estimate the orbital period of the companion provided that

$\Delta r_{a r m}, V_{w}\left(r_{a r m}\right)$, and $c_{\mathrm{s}}\left(r_{a r m}\right)$ are known from multi-wavelength observations. Alternatively, it can be rewritten as

$$
\Delta r_{a r m}=\frac{2 \pi\left(V_{w}+c_{\mathrm{s}}\right)}{\left(G M_{*}\right)^{1 / 2}} \times r_{p}^{3 / 2},
$$

which can be used to determine the orbital distance of the companion provided that knowledge of the stellar mass $M_{*}$ is obtained separately.

The application of our results to the analysis of observation data is somewhat qualitative given that there are many uncertainties in the inner part of the envelopes resulting from the effects associated with the detailed driving of the stellar wind by stellar radiation, pulsation of the AGB star, the gas heating-cooling properties, and the accretion properties of the substellar object. Nevertheless our results suggest that the search for spiral arm patterns in the circumstellar envelope of an AGB star is desirable in order to make use of the full potential of the probes described in this paper to infer the presence and to constrain the properties of unseen objects orbiting about evolved mass-losing stars. 


\section{ACKNOWLEDGMENTS}

This research is supported by the Theoretical Institute for Advanced Research in Astrophysics (TIARA) in the Academia Sinica Institute of Astronomy and Astrophysics (ASIAA). We would like to thank the organizing committee of the workshop on Planetary Systems Beyond the Main Sequence for encouraging the presentation as well as the workshop participants for valuable discussion. H.K. is grateful to Paul M. Ricker for advice on computational issues.

\section{REFERENCES}

Aikawa, Y., \& Herbst, E. 1999, A\&A, 351, 233

Alibert, Y., Mordasini, C., Benz, W., \& Winisdoerffer, C. 2005, A\&A, 434, 343

Bondi, H., \& Hoyle, F. 1944, MNRAS, 104, 273

Edgar, R. 2004, New Astronomy Reviews, 48, 843

Fong, D., Meixner, M., Sutton, E. C., Zalucha, A., \& Welch, W. J. 2006, ApJ, 652, 1626

Gail, H.-P., \& Sedlmayr, E. 1987, A\&A, 177, 186

Goldreich, P., \& Tremaine, S. 1979, ApJ, 233, 857

Hayashi, C. 1981, Progress of Theoretical Physics Supplement, 70, 35

Hubickyj, O., Bodenheimer, P., \& Lissauer, J. J. 2005, Icarus, 179, 415

Ikoma, M., Nakazawa, K., \& Emori, H. 2000, ApJ, 537, 1013

Kim, H., \& Kim, W.-T. 2007, ApJ, 665, 432

Kim, H., Kim, W.-T., \& Sánchez-Salcedo, F. J. 2008, ApJ, 679, L33

Kim, H., \& Taam, R. E. 2011, in prep.

Lamers, H. J. G. L. M., \& Cassinelli, J. P. 1999, Introduction to Stellar Winds, Cambridge University Press, Cambridge, UK

Masset, F. S. 2008, EAS Publications Series, 29, 165

Mauron, N., \& Huggins, P. J. 2006, A\&A, 452, 257

Morris, M., Sahai, R., Matthews, K., Cheng, J., Lu, J., Claussen, M., \& Sánchez-Contreras, C. 2006, Planetary Nebulae in our Galaxy and Beyond, 234, 469

Ostriker, E. C. 1999, ApJ, 513, 252

Parker, E. N. 1958, ApJ, 128, 664

Winters, J. M., Le Bertre, T., Jeong, K. S., Helling, C., \& Sedlmayr, E. 2000, A\&A, 361, 641 